\title{
Monte-Carlo simulations of the recombination dynamics in porous silicon
}

\author{
H. Eduardo Roman \\ Institut für Theoretische Physik, Universität Giessen, Heinrich-Buff-Ring 16, D-35392 Giessen, \\ Germany \\ and Lorenzo Pavesi* \\ I.N.F.M. and Dipartimento di Fisica, Università di Trento, via Sommarive 14, I-38050 Povo \\ (Trento), Italy.
}

(received October 29, 2018)

\begin{abstract}
A simple lattice model describing the recombination dynamics in visible light emitting porous Silicon is presented. In the model, each occupied lattice site represents a Si crystal of nanometer size. The disordered structure of porous Silicon is modeled by modified random percolation networks in two and three dimensions. Both correlated (excitons) and uncorrelated electronhole pairs have been studied. Radiative and non-radiative processes as well as hopping between nearest neighbor occupied sites are taken into account. By means of extensive Monte-Carlo simulations, we show that the recombination dynamics in porous Silicon is due to a dispersive diffusion of excitons in a disordered arrangement of interconnected Si quantum dots. The simulated luminescence decay for the excitons shows a stretched exponential lineshape while for uncorrelated electron-hole pairs a power law decay is suggested. Our results successfully account for the recombination dynamics recently observed in the experiments. The present model is a prototype for a larger class of
\end{abstract}


models describing diffusion of particles in a complex disordered system.

Pacs: 71.55.Jv;72.20.jv;78.47.+p;61.43.Bn 


\section{INTRODUCTION}

Silicon, the most studied semiconductor, is not a good light emitter, especially in the visible range, because of its indirect band gap transition in the infrared $(1.1 \mathrm{eV})$. The recently discovered optical properties of porous forms of Silicon ( $\mathrm{p}$-Si) have therefore attracted considerable interest. [1 4 In addition to the internal nanometer sized structures which are distributed in space according to a complex topology, [5] these new materials have similar spectral behaviors, the most prominent are a rather high luminescence quantum efficiency and an unexpected wide range of luminescence lifetimes (ranging from microseconds to milliseconds), depending on temperature.

The mechanism of the luminescence emission is still unclear. Three main models have been proposed and survived to the recent discussions:

1. quantum recombination model, 6 8 8

2. surface state model, [9]

3. molecular recombination model. 10 12

The first two models agree on the fact that the energy spectrum of $\mathrm{p}-\mathrm{Si}$ is a result of the quantum confinement of carriers in Si nano-crystals. Still under debate, is the shape of the nano-crystal that has been described either in terms of "undulating" quantum wires [6] or quantum dots. [8] The quantum recombination and the surface state models differ in their predictions about the origin of the luminescence. In the first case, the luminescence is due to confined and localized excitons, while in the second, the luminescence is due to electron-hole pair recombination, where the electron and the hole could be found either in a bulk nano-crystal state ('extended' state), or being trapped into a surface nano-crystal state ('localized' state). The third model, the molecular recombination model, considers that some particular molecular species like polysilane chains (e. g. SiH chain [10]) or siloxene-like rings

(Si-O-H) [1] form on the surface of the Si nano-crystals. The luminescence is due to the carrier trapping into these species. 
Recent experiments [13 18,?,20] have provided clear evidence of anomalous relaxation behavior of the luminescence. The decay lineshape, for a single observation energy, is not described by a single exponential function. This non-exponential behavior can be commonly described by a stretched exponential function, 21 23 defined as

$$
\mathrm{L}(\mathrm{t})=\mathrm{L}(0) \exp \left[-(\mathrm{t} / \tau)^{\beta}\right]
$$

where $\mathrm{L}(\mathrm{t})$ is the time dependent luminescence intensity, $\tau$ is a lifetime and $\beta \leq 1$ a dispersion exponent. In general, values of $\beta<1$ correspond to the existence of a broad distribution of lifetimes. In some circumstances, such broad distributions may be the result of a diffusive motion of the excited carriers. This may be the case in p-Si, as suggested recently in terms of trap-controlled hopping processes. [18] We elaborate this idea further in this work.

Motivated by these experimental results, and by the fact that little is known about the recombination dynamics of the charge carriers in these materials, we have initiated a detailed numerical study of the underlying transport behavior in p-Si by means of Monte-Carlo (MC) simulations.

In this work, we present a simple model of p-Si in which nanometer sized crystals (nanocrystals), characterized by a distribution of radiative and non-radiative recombination times, are assumed to be randomly placed at the sites of percolation-like clusters defined on square and simple cubic lattices. Charge carriers are allowed to hop between nearest-neighbor occupied cluster sites. The competing effect between radiative and non-radiative transitions in a single Si nano-crystal, as well as the effects of geometrical constraints on hopping of carriers due to the complex topology of percolation clusters, are discussed.

Even though our simulations are based on the quantum recombination model, [6, [7] they are consistent with other possible recombination mechanisms because the model concentrates on the dynamics of the recombination and not on the recombination process itself. However, we found that most of the data are better explained by our simulations when the quantum recombination model is assumed. In addition, since our model is based on quite general assumptions about the geometrical disorder involved, we expect that it may be applied to 
a larger class of physical systems characterized by both a structural and local disorder, of which p-Si is just one possible example.

The paper is organized as follows: in Section II the model is described, and exact solutions obtained in the case of isolated Si nano-crystals are discussed. In Sections III and IV, simulation results for one-, and two- and three-dimensional systems, respectively, are presented. In Section V, a discussion of the present results is presented and compared to other results already known in the literature. Finally, in Section VI we give some concluding remarks.

\section{MODEL FOR A SI NANOMETER SIZED CRYSTALS}

We consider a lattice model for p-Si in which occupied and empty sites are present. Each occupied site of the lattice represents a Si nano-crystal. In one dimension, all the sites are occupied by Si nano-crystals, while in higher dimensions the occupied sites are randomly distributed in space and interconnected to each other according to the topology of percolation-like clusters (see Section IV). We further assume that the physical properties, relevant for our problem, of such Si nano-crystals are suitably described in terms of quantum dots (QD), for which many theoretical results are presently available.

\section{A. The size and radiative energy of a Si nano-crystal}

Each Si nano-crystal, or equivalently, each occupied site of the lattice, is characterized by a size $d$, representing its mean diameter (typically in the range 15 to $55 \AA$ [24]). Since the actual spatial distribution of nano-crystal sizes is not known a priori, the sizes $d_{i}$ at site $i$ are chosen randomly according to a Gaussian distribution $P(d)$. [25] We used $P(d) \sim$ $\left(1 / \sigma_{d}\right) \exp \left[-\left(1 / 2 \sigma_{d}^{2}\right)\left(d-d_{0}\right)^{2}\right]$, centered at $d_{0}=32.4 \AA$ and having a width $\sigma_{d}=0.8 \AA$.

In one dimension, this local randomness is the only source of disorder in the model, while in higher dimensions, an additional (geometrical) disorder, due to the complex structure of percolation-like clusters, is also present (see Section IV). 
Due to the experimental method of preparation of p-Si, however, some correlation between the sizes $d$ may actually be present for nearby Si nano-crystals, from which one may reasonably expect that strong local variations in $d$ are not likely to occur. [5] To take this effect into account in a simple way, the sizes $d$ corresponding to nearest neighbor occupied sites are not allowed to differ by more than a prefixed amount. We are going to specify this point below.

By neglecting the excitonic binding energy, the optical band-gap energy $E$ associated to a given Si nano-crystal is assumed to correspond to the emission energy determined experimentally. $E_{i}$ is related to the size $d_{i}$ through the power-law relation recently suggested by calculations performed for spherical quantum dots. [26] Based on these calculations, we assume $E_{i}=E_{0}\left(d_{0} / d_{i}\right)^{n}$, with $n \cong 1.39$, where $E_{0}=1.86 \mathrm{eV}$ is the transition energy for a Si nano-crystal of size $d_{0}$. The corresponding distribution for $E$ is then of the form,

$$
P(E) \sim \frac{1}{\sigma_{d}} \frac{1}{E^{1+1 / n}} \exp \left[-\left(d_{0}^{2} / 2 \sigma_{d}^{2}\right)\left(\left(E_{0} / E\right)^{1 / n}-1\right)^{2}\right]
$$

centered around $E_{0}$ and having a width at half-maximum of about $0.16 \mathrm{eV}$ (see Fig. 11).

It is instructive to obtain estimates of the total density of states $\rho(\epsilon)$ resulting from these distributions. For simplicity, an ensemble of quantum dots of cubic shape and side $L_{x}=L_{y}=L_{z}=d$ is considered. [27] The (confined) electronic states have energies $\epsilon\left(n_{x, y, z}\right)=$ $\epsilon_{0}\left(n_{x}+n_{y}+n_{z}\right)$, where $\epsilon_{0}=\hbar^{2} \pi /\left(2 \mu d^{2}\right), n_{x}, n_{y}$ and $n_{z}$ are the principal quantum numbers for motion in the $x, y$ and $z$ directions, $n_{x, y, z} \geq 1$ and $\mu$ is the effective mass. The transition energy $E$ can be related to the ground state energy of the QD by $E=3 \epsilon_{0}+E_{\mathrm{Bulk}}$, where $E_{\mathrm{Bulk}}=1.15 \mathrm{eV}$ is the bulk-Si indirect gap. The density of states for a single quantum dot of size $d$ with an energy barrier of height $\mathrm{V}(\mathrm{QD})$ is then 27]

$$
\rho_{d}(\epsilon) \cong \frac{2 V(\mathrm{QD})}{d^{3}} \sum_{n_{x, y, z}} \delta\left(\epsilon-\epsilon\left(n_{x, y, z}\right)-E_{\mathrm{Bulk}}\right),
$$

where $\epsilon$ is an energy and the total density of states is

$$
\rho(\epsilon)=\sum_{d} P(d) \rho_{d}(\epsilon)
$$


This quantity is plotted in Fig. 11 assuming a Gaussian broadening of the single QD state of $0.01 \mathrm{eV}$. The density-of-states of an ordered ensemble of QDs should reveal a series of distinct peaks corresponding to the different confined states $\epsilon\left(n_{x, y, z}\right)$ in the QD. The random Gaussian distribution of sizes, i. e. E, smears out the discrete peaks and yields an approximately exponential behavior of the form $\rho(\epsilon) \sim \exp \left(\epsilon / \epsilon_{a}\right)$, with $\epsilon_{a} \cong 0.35 \mathrm{eV}$, at large $\epsilon$.

To deal with the above mentioned spatial correlations in $d$, the difference in energy between nearest-neighbor sites $i$ and $j, \Delta E_{i j}=E_{j}-E_{i}$, is allowed to take absolute values smaller than a given cutoff $E_{\text {cut }}$. This is the criterion employed to accept a given value of $d_{j}$, with respect to the previous value at site $i$, from the Gaussian distribution. In dimensions higher than one, this criterion is applied simultaneously with the growing process (see Section IV). For convenience, we consider variations in energy, rather than variations in size to select the values of the local quantities $E$ and $d$. The results are not sensitive to this choice. Typical calculations have been performed for values in the range $10^{-4}<E_{\text {cut }}<0.4 \mathrm{eV}$ (see Section III). $E_{\text {cut }}$ is the first parameter in the model.

\section{B. The radiative and non-radiative recombination times}

Within each Si nano-crystal, both radiative and non-radiative recombination processes

occur when excited electron-hole pairs are present. Both processes depend on the size $d$ of the nano-crystal.

The emission process is assumed to be due to excitonic recombinations in a QD. In this case, it was shown that the radiative recombination time, $\tau_{\text {rad }}$, results from the thermal balance between the occupation of the exchange splitted singlet and triplet excitonic states, |7]

$$
\tau_{\text {rad }}(E, T)=\tau_{\text {tripl }}\left[\frac{1+(1 / 3) \exp \left(-\Delta E_{x} / k_{B} T\right)}{1+(1 / 3)\left(\tau_{\text {tripl }} / \tau_{\text {sing }}\right) \exp \left(-\Delta E_{x} / k_{B} T\right)}\right] .
$$

$\tau_{\text {sing }}$ (the radiative lifetime for the singlet excitonic state) is obtained from the values calculated in Ref. [28], 


$$
\tau_{\text {sing }}(E)=\tau_{\text {sing }}^{(0)}\left(\frac{E_{\text {Bulk }}}{E-E_{\text {Bulk }}}\right)^{n_{s}}
$$

where $n_{s}=1.5$, and $\tau_{\text {sing }}^{(0)}$ is the second parameter in the model. At the present, no theoretical calculations are available for the radiative lifetime of the triplet excitonic state, $\tau_{\text {tripl }}$. Thus, $\tau_{\text {tripl }}$ is estimated from the experimental data reported in Ref. [7],

$$
\tau_{\text {tripl }}(E)=\tau_{\text {tripl }}^{(0)}\left[\tau_{\text {sing }}(E)\right]^{\alpha_{s}}
$$

where $\tau_{\text {tripl }}^{(0)}=2368 \mu \mathrm{s}$, and $\alpha_{s}=0.307$.

Finally, the quantum confinement enhanced exchange energy $\Delta E_{x}=E_{x}^{S i}\left(d_{x} / d\right)^{3}$, where $E_{x}^{S i}=3.165 \times 10^{-4} \mathrm{eV}$ is the exchange energy in crystalline $\mathrm{Si}$ and $d_{x}=43 \AA$, is obtained from the calculations in Ref. [29].

Non-radiative recombinations have been considered as multiphonon transition processes for trapping on a deep center, [30] for which the non-radiative recombination time, $\tau_{\mathrm{nr}}$, is given by

$$
\tau_{\mathrm{nr}}(T)=\tau_{\mathrm{nr}}^{(0)} \frac{e^{2 n_{T} S}}{\left(n_{T}+1\right)^{p}}
$$

where $n_{T}=\left[\exp \left(\hbar \omega_{p h} / k_{B} T\right)-1\right]^{-1}$ is the density of phonons with energy $\hbar \omega_{p h}$, and $S$ and $p$ are related to the details of the capturing center. Here we have used nano-crystal size independent quantities, with $\hbar \omega_{p h} / k_{B}=800 \mathrm{~K}, S=1$, and $p=25$. If bulk-Si optical phonons are involved, $\hbar \omega_{p h} / k_{B} \simeq 1100 \mathrm{~K}$; lower values can be justified by the typical softening of the phonon modes in a confined system on a localized center. $\tau_{\mathrm{nr}}^{(0)}$ is the third parameter in the model.

It should be emphasized that the actual nature and origin of non-radiative phonon transition processes in p-Si nano-crystals remain to be understood. Here, such processes are included because they certainly play a very important role in the recombination dynamics of carriers. [8,9] The form for $\tau_{\text {nr }}$ in Eq. 8 can be only considered as a first approximation to the actual form of the non-radiative recombination time, yet it is expected to describe the physics involved correctly, at least in a qualitative fashion. In this work, the same $\tau_{\text {nr }}$ has been employed for all the Si nano-crystals. 


\section{The hopping rates between Si nano-crystals}

In addition to the internal structure of Si nano-crystals, hopping of electrons, holes and excitons, may actually occur between nearby nano-crystals. To describe the diffusion of carriers in p-Si, we consider hopping processes only between nearest neighbor (n.n.) occupied sites of the lattice for two different models. In the first model, only one type of carrier hops, representing the exciton, while in the second, two different types of carriers can hop, representing the electron and hole. If the electron and hole (e-h) motions are strongly correlated, e.g. if the hole closely follows the electron as it moves in the system, one has essentially the first model. As we will see in Sections III and IV, a qualitatively different behavior is obtained when e-h hops are completely uncorrelated. In both models, correlated and uncorrelated e-h pairs, the on site radiative recombination is assumed to be excitonic.

The transition rates for a carrier of type $x(x \equiv e x$, representing the exciton, $x \equiv e$, the electron, and $x \equiv h$, the hole) from the site $i$ to its nearest-neighbor occupied site $j$ is defined as

$$
P_{i j}^{x}=\frac{1}{\tau_{\text {hop }}^{x}} \quad \text { when } \quad E_{j}-E_{i} \leq 0
$$

and

$$
P_{i j}^{x}=\frac{1}{\tau_{\text {hop }}^{x}} \exp \left(-f_{x} \Delta E_{i j} / k_{B} T\right) \quad \text { when } \quad E_{j}-E_{i} \geq 0
$$

where $\Delta E_{i j}=E_{j}-E_{i}$. When the site $j$ is not occupied, as it may occur in two- and three-dimensional systems, then $P_{i j}^{x}=0$. Here, $0<f_{x} \leq 1$ is an additional parameter

related to the band structure of nano-crystals, and $\tau_{\text {hop }}^{x}$ is a characteristic time for tunneling of $x$-carriers between nearby (n.n.) Si nano-crystals, which is temperature independent. In Eqs. 9 and 10, no dependence on the intersite distance, $r_{i j}$, between n.n. Si nano-crystals is explicitly shown. In a more refined version of the present model, one could consider in Eqs. 9 and 10 a dependence on $r_{i j}$ of the form

$$
\frac{1}{\tau_{\text {hop }}^{x}}=\nu_{x} \exp \left(-\gamma_{i j} r_{i j}\right)
$$


where $\nu_{x}$ is an attempting frequency and $\gamma_{i j}$ is related to the energy barrier which separates the QDs at site $i$ and $j$. The quantities $\gamma_{i j}$ and $r_{i j}$ may then be considered as random variables. For simplicity, we use $\tau_{\text {hop }}^{x}=\tau_{\text {hop }}$ here, independently of the type of carrier. Thus, the dependence of hopping rates on $x$ is only contained in the factor $f_{x}$.

In our calculations, we have arbitrarily chosen $f_{e}=1 / 3, f_{h}=2 / 3$ and $f_{e x}=0.4$ in order to take into account the distribution of the energy-gap discontinuity, $\mathrm{E}-\mathrm{E}_{\mathrm{Bulk}}$, among the valence and conduction bands. Extensive Monte-Carlo simulations have been done by using the same set of parameters but for different $f_{e x}$. The effect of variing $f_{e x}$ is to change the barrier for hopping, $f_{e x} \Delta E_{i j}$, which is mostly relevant at low temperatures. By increasing $f_{e x}$, a decrease in the luminescence decay rate occurs, but essentially no changes in the luminescence decay lineshapes are observed, i. e. in Eq. 目 $\tau$ increases and $\beta$ remains constant. The mean distances over which the excitons move before recombination are reduced as the effective barrier, $f_{e x} \Delta E_{i j}$, is increased. However, for $f_{e x}$ in the interval $[0.2-0.6]$ the numerical results are qualitatively not affected and quantitatively they change by less than $10 \%$.

Before going on with the discussion of the results, we briefly summarize the free parameters entering the model:

(1) $E_{\text {cut }}$, absolute energy difference cut-off of nearby occupied sites in the lattice.

(2) $\tau_{\text {sing }}^{(0)}$, prefactor for the singlet radiative recombination time (Eq. 6).

(3) $\tau_{\mathrm{nr}}^{(0)}$, prefactor for the non-radiative recombination time (Eq. $\mathrm{Q}$ ).

(4) $\tau_{\text {hop }}$, prefactor for the tunneling of carriers between nearest-neighbor occupied sites (Eq. 9 and (10).

Table I reports the values of all the parameters used in our simulations for $1 \mathrm{D}, 2 \mathrm{D}$ and 3D systems whose results are presented in the following. 


\section{The case of isolated Si nano-crystals}

Let us consider the case in which no hopping between n.n. Si nano-crystals occurs in the system. This limiting situation may well describe p-Si samples in which Si nano-crystals are efficiently passivated and surrounded by thick surface oxide layers. This case represents an ensemble of isolated QDs, each characterized by its own size $d$ and transition energy E. To obtain the luminescence spectrum of the whole ensemble as a function of time, for different

observation energies and temperatures, the rate equation describing the time evolution of the density $N(t)$ for a single carrier at a given Si nano-crystal (site) is solved. Such a rate equation reads,

$$
\frac{d N(t)}{d t}=-\frac{N}{\tau_{\mathrm{rad}}}-\frac{N}{\tau_{\mathrm{nr}}}
$$

and the exact solution is

$$
N(t)=N(0) \exp (-t / \tau) \quad \text { with } \quad \frac{1}{\tau}=\frac{1}{\tau_{\mathrm{rad}}}+\frac{1}{\tau_{\mathrm{nr}}} .
$$

Typical behaviors of the different times entering in Eq. 13 as a function of temperature, for fixed energy $E$, are shown in Fig. 2.

The luminescence $L(t)$ from a single Si nano-crystal is just $L(t)=N(t) / \tau_{\text {rad }}$, i.e.

$$
L(t)=L(0) \exp (-t / \tau)
$$

yielding simple exponential decay, i.e. $\beta=1$ (cfr. Eq. 11). As pointed out in Ref. [8], the luminescence decay time is essentially determined by non-radiative recombinations at room temperature. According to Eq. 13 and Fig. 2, non-radiative recombinations play a main role also at low temperatures when the radiative lifetime, dominated by the triplet recombination lifetime, becomes very large. In the intermediate temperature range, when the quantum efficiency also increases, the singlet lifetime may dominate the recombination lifetime, if it becomes sufficiently small and thermal quenching of the phonon population takes place, decreasing the capture cross-section to the non-radiative recombination centers. 
This is case 3 in Fig. 2. At sufficiently high temperatures, non-radiative recombinations dominate again.

The total radiated intensity $I(E, T)=\int_{0}^{\infty} d t L(t)=N(0) \tau / \tau_{\text {rad }}$, is related to the corresponding quantum efficiency, $\epsilon(E, T)$ defined as

$$
\epsilon(E, T) \equiv \frac{I(E, T)}{N(0)}=\frac{\tau_{\mathrm{nr}}}{\tau_{\mathrm{nr}}+\tau_{\mathrm{rad}}} .
$$

For illustration, the quantity $\epsilon(E, T) P(E)$, representing the quantum efficiency of the ensemble of QDs described by Eq. 2 for an energy E, is plotted for several temperatures versus energy in Fig. 3. The total quantum efficiency of the ensemble, $\epsilon(T)$, is just the sum of Eq. 15 over the total number of Si nano-crystals in the system, i.e. $\epsilon(T)=\sum_{E} P(E) \epsilon(E, T)$. This quantity is shown in Fig. 世. At high temperatures, we find an exponential behavior $\epsilon(T) \sim \exp \left(-T / T_{0}\right)$, where $T_{0} \cong 59 \mathrm{~K}$ (see cases (1) and (2) in Fig. 4), in good agreement with recent experimental results (see e.g. Ref. [31]). The value $\hbar \omega_{p h} / k_{B}=800 \mathrm{~K}$ and $p=25$ (see Section II.B), were chosen such that $\epsilon(T)$ displays a maximum in the range $100 \mathrm{~K}<T<200 \mathrm{~K}$ and a value of about $10 \%$ at room temperature, as is experimentally observed. 8]

Finally, the absorption coefficient $\alpha(E)$ of the ensemble can be obtained from the relation

$$
\alpha(E, T) \simeq \frac{\text { const }}{E}|M|^{2} \rho(E)
$$

where $|M|$ is the dipole matrix element at the band-edge describing radiative transitions in the $\mathrm{QD}$ and $\rho(E)$ is the total density of states. $|M|$ is related to the oscillator strength of the transition and hence to the radiative lifetime, i.e. $|M|^{2} \sim \tau_{\mathrm{rad}}^{-1}(E, T)$. 28 Using

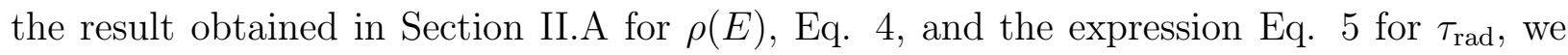
obtain the absorption coefficient of the ensemble as displayed, for various temperatures, in Fig. 5. Remarkably, this very simple model is able to reproduce the main features of the measured absorption coefficient, [9] i.e. a quite sharp edge at about $1.6 \mathrm{eV}$ and an increasing exponential shape at high energies with a slope which depends on the temperature. An exponential fit at high energies, $\alpha(E) \sim \exp \left(E / E_{0}\right)$, of the curves shown in the figure yields 
for the energy slopes: $E_{0}=0.55 \mathrm{eV}$ for $T=30 \mathrm{~K}, 0.34 \mathrm{eV}$ for $T=100 \mathrm{~K}, 0.29 \mathrm{eV}$ for $T=200 \mathrm{~K}$ and $0.28 \mathrm{eV}$ for $T=300 \mathrm{~K}$. The temperature dependence of $\alpha(E)$ is due to the $\tau_{\text {rad }}$ term in Eq. 16. The exponential dependence of the absorption coefficient has been repetitively observed in p-Si [31,32] and attributed to an Urbach-like edge as in amorphous Silicon. The origin of the Urbach-tail is not precisely known so far but it is believed to be due to disorder. In the present model, it results from the overlap of the 0-dimensional densities of states associated to the QDs randomly arranged in space.

\section{E. Rules for the Monte-Carlo simulations}

In the case in which hopping of carriers between nearby Si nano-crystals takes place, the rate equation for $N(t)$, Eq. 12, is modified to

$$
\frac{d N_{i}(t)}{d t}=-\frac{N_{i}}{\tau_{\mathrm{rad}}}-\frac{N_{i}}{\tau_{\mathrm{nr}}}-\sum_{j} P_{i j} N_{i}(t)+\sum_{j} P_{j i} N_{j}(t)
$$

yielding a system of coupled differential equations for the different occupied sites $i$. For simplicity, we have omitted the index $x$, denoting the type of carrier, from Eq. 17. The third term in Eq. 17 represents the outgoing particles from site $i$ (loss term), while the last term the incoming particles to site $i$ from the neighbor sites $j$ (feeding term). In one dimension, the number of nearest neighbor sites $n=2$, and because of structural disorder, in two dimensions $1 \leq n \leq 4$, and in three dimensions $1 \leq n \leq 6$. The system in Eq. 17 is conveniently solved by Monte-Carlo (MC) simulations when the total number of effectively coupled occupied sites $i$ becomes large. In particular, certain constraints for the occurrence of recombination processes, such as the simultaneous presence of an electron and a hole at a given site, can be easily implemented with the MC method.

Before discussing the MC-rules, we need to determine the unit of time, denoted as $\tau_{0}$, which should be sufficiently small such that faster transition events are well described. Once the transition times $\tau$ have all been determined in the system, one can take $\tau_{0}=\tau_{\text {fast }} / n_{t}$, where $\tau_{\text {fast }}$ is the smallest transition time in the ensemble and $n_{t}>1$. We have used $n_{t}=10$ 
in our simulations. Notice that the time $t$ becomes now a discrete variable, i.e. $t=n \tau_{0}$, where $n \geq 1$ denotes the $n$th MC-step. The total elapsed time for each MC-step is just $\tau_{0}$.

We can now discuss the MC-rules for the present model. Since we are interested only in the case of very low carrier density, i.e. different electron-hole pairs in the system do not see each other (no interaction effects or Auger recombinations), we study the time evolution (trajectory) of a single electron-hole pair (either as an exciton or as two independent particles), which is initially located $(t=0)$ at the center of the lattice. Averages are then performed over many trajectories and different realizations of disorder.

During the $n$th MC-step, a particle at site $i$ may undergo one of the following four different processes:

(1) decaying radiatively (annihilate) with a probability $p_{\text {rad }}=\tau_{0} / \tau_{\text {rad }}$,

(2) decaying non-radiatively (annihilate) with a probability $p_{\mathrm{nr}}=\tau_{0} / \tau_{\mathrm{nr}}$,

(3) hopping to a n.n. site $j$ with a probability $p_{i j}=\tau_{0} P_{i j}$, or

(4) remaining at site $i$ with a probability $p_{\text {sit }}=1-p_{\text {rad }}-p_{\mathrm{nr}}-\sum_{j} p_{i j}$.

In the case in which an electron and a hole are considered, a decay can only take place when both carriers are located at the same site.

According to our definition of $\tau_{0}$, all the above probabilities are smaller than one as required. To decide which event will take place for a given particle at site $i$, we generate a random number $r$, uniformly distributed in the range $0<r<1$. Then, we evaluate the partial sums over the probabilities, i.e. $\sum_{k=1}^{m} p_{k}=S_{m}$, where the index $k=1$ represents a radiative recombination event, $k=2$ a non-radiative one, etc., and $m \geq 1$. The successful event is the one for which $S_{m}>r$ for the first time. For example, consider a 1D system and let $p_{\mathrm{rad}}=p_{\mathrm{nr}}=0.2$ (equally likely radiative and non-radiative recombinations) and $p_{i(i-1)}=p_{i(i+1)}=0.1$ (i.e. equal hopping rates to two n.n. sites). Then, if $r=0.55$, the particle will undergo the fourth possibility, i.e. it will hop to the second n.n. site. If, however, $r>0.6$, the particle will remain sit at its present site.

A single MC-step is completed when the above discussed procedure has been applied to each particle in the system (either the exciton, or the electron and hole), and the time 
$t$ is increased by $\tau_{0}, t=t+\tau_{0}$. At the $n$th MC-step, corresponding to time $t=n \tau_{0}$, the luminescence of the system is simply obtained by counting the total number of radiative events during the $n$th MC-step. Since we are also interested in the energy dependence of the luminescence, those radiative events originated at sites characterized by transition energies $E^{\prime}$ close to the observation energy $E$ (i.e. $\left|E^{\prime}-E\right|>\delta E$ ) are recorded separately. In our simulations we used $\delta E=0.01 \mathrm{eV}$.

We proceed with the numerical results obtained for one-dimensional lattices.

\section{RESULTS FOR ONE-DIMENSIONAL LATTICES}

At very high porosities, p-Si is characterized by a filamentary structure having a quasione dimensional character on small length scales. 333 Thus, we start our discussion about transport (diffusion) of carriers in p-Si on a simplified one-dimensional model. Preliminary results with the set of parameters named case (3) in Figs. 2 and [t are reported in Ref. [19].

When the carriers are allowed to hop between n.n. sites of the linear lattice, the diffusive motion takes place in a highly irregular energy landscape. This feature of the model is illustrated in Fig. 6, where the local transition energies $E$ are plotted as a function of position on the one-dimensional lattice. In the figure, three cases are considered for selected values of the parameter $E_{\text {cut }}$ (see Section II.A). Although local variations in energy can be smoothed out when $E_{\text {cut }}$ becomes sufficiently small, on large length scales appreciable variations of $E$ will always occur (see Fig. 6). This has important consequences when considering hopping processes, because carriers must eventually overcome an effective large barrier to diffuse out of a region of local minimum energy. According to our definition of the transition rates in Eq. 10, thermally activated hopping against such large scales effective barriers will be strongly hindered at sufficiently low temperatures, even in the case of low $E_{\text {cut }}$ values. The low energy sites with low $p_{\text {rad }}$ and high energy barriers $\Delta E_{i j}$ act as temporary traps. Excitons which relax to these sites are temporary trapped into them and have to wait long times before being released. 
To quantify the effect of disorder on diffusion we calculate the mean square displacement of the carrier as a function of time, $R^{2} \equiv\left\langle r^{2}(t)\right\rangle-\langle\vec{r}(t)\rangle^{2}$, and investigate its temperature dependence. $\vec{r}(t)$ is the displacement with respect to the initial position, which is assumed to be the origin. To study the effect of the dynamics of carriers on the time dependence of $L(t)$, we have solved Eq. 17 for the values given in the Table I. Since in experiments all sites of the system are homogeneously excited at $t=0$, including those with energy $E$ close to the observation energy $E_{\text {obs }}$, initially, the electron-hole pair is placed at a site (the center of the lattice) characterized by an energy $E \cong E_{\text {obs }}$.

In the case of excitons, the behavior of the luminescence is shown in Fig. 7 for four different temperatures and observation energy $E_{\mathrm{obs}}=1.86 \mathrm{eV}$. Two remarks are evident: i) the decay departs from the simple exponential decay obtained for isolated QDs (corresponding to $\tau_{\text {hop }}=\infty$ ), and ii) the time scale of the decay is strongly temperature dependent. The last item is due to the thermal population of the singlet state (fast radiative recombination time) and to the onset of efficient non-radiative decay, especially at the highest temperature. The lineshape analysis shown in Fig. 8 evidences that a stretched exponential function is required to fit the decay. The single exponential or the double exponential functions are able to fit the decay only on a limited time range.

The stretched exponential decay reflects the fact that a time delay between recombination events at a given QD may occur when the exciton can diffuse out of the QD. Such time delay can persist on large time scales since the exciton may either diffuse far away from the site at which the radiative event is expected, or may become temporarily trapped at a nearby site with a lower transition energy. When the observed radiative events are collected from sites with similar transition energies $E$, the random local environment around each of these sites leads to a distribution of recombination times, which are in general different than the single value $\tau_{\text {rad }}(E, T)$ for that energy $E$. This dispersion of recombination times causes the stretched exponential behavior observed in the model.

In the case of uncorrelated electron and hole motion, a quite different behavior is observed (see Fig. 9). At high temperatures, and intermediate times, an approximate power-law decay 
of the form

$$
L(t) \sim t^{-\alpha}
$$

takes place. The range of times over which the power-law decay seems to occur grows as the temperature is raised. Here, the uncorrelated motion of the electron and hole leads to a dramatic slowing down of the luminescence decay, since the two carriers are no longer constrained to be all the time simultaneously at the same site. Similar lineshapes have been observed for amorphous hydrogenated Silicon. [34 In this system, the luminescence is explained as due to geminate recombination between e-h pair localized in band tail states. 35

We have performed extensive MC simulations for different temperatures. In the case of excitons, the decay lineshapes are fitted by a stretched exponential function for all the temperatures considered. A statistical weigth of the simulation points has been used to obtain the best fit function. The results of the fits are summarized in the upper panel of Fig. 10. Typical errors for $\tau$ of $10 \%$ and $\beta$ of 0.02 are expected, due to statistical fluctuations and goodness of the fits. The lifetime $\tau$ decreases significantly as the temperature is raised, while the dispersion exponent $\beta$ increases and tends to unity at high temperatures.

We have also calculated the quantum efficiency $\epsilon(E, T)$ as a function of $E$ for a fixed temperature, displaying a maximum around $E=1.86 \mathrm{eV}$ and found that hopping of carriers does not affect sensitively $\epsilon(E, T)$. This is clear because $\epsilon(E, T)$ is essentially determined by the on-site recombination dynamics. Independently of the dispersive motion, all the carriers recombine radiatively or non-radiatively after a sufficiently long time. The diffusion only influences the recombination dynamics by introducing long lived recombinations.

In Fig. 11, we show the values of $R^{2}(t)$ for different temperatures. At intermediate and high temperatures, an anomalous behavior of the form $R^{2}(t) \sim t^{2 / d_{w}}$ with $d_{w} \geq 2$, takes place. Such anomalies in $R^{2}(t)$ are typical of diffusion on fractals, where the presence of dangling ends and loops on all length scales in the fractal slows down the diffusion process on all time scales. On fractals, however, the diffusion exponent $d_{w}$ is temperature independent, 
[36] while here $d_{w}$ turns out to depend strongly on temperature (see Fig. 12). The anomalies in $R^{2}$ are due to the random distribution of hopping rates, which is known to yield values of $d_{w}>2$ and dependent on temperature (see e.g. Ref. [37]). At low temperatures, however, the time dependence of $R^{2}(t)$ departs from a power law.

At sufficiently low temperatures, indeed, the present model is expected to display transport behavior similar to diffusion in the presence of random fields (Sinai model), see Refs. [38] and [39. To show this, we have drawn in Fig. 13 a small section of a typical energy landscape versus position. The arrows represent the local fields felt by the carrier which is proportional to the energy difference $\Delta E_{i j}$ between the two n.n. sites $i$ and $j$. Due to the assumed random distribution of nano-crystal sizes in the system, the local fields are randomly oriented, similarly as in the Sinai model. Diffusion of carriers in the system is biased by these random fields and becomes ultra-anomalously slow. Of course, in the present model the energy barriers (and also the potential valleys) can not grow indefinitely as in the Sinai case. However, when the temperature is sufficiently low, diffusion is strongly hindered and the carriers can only explore small length scales since the effect of the random fields becomes dominant. In these circumstances, a logarithmic time dependence is expected

$$
R^{2}(t) \sim(\log t)^{4}
$$

at long times. Results obtained for the case of excitons are plotted in Fig. 14. These suggest that for large times and low temperatures, our model displays logarithmic time dependencies of the form predicted by Eq. 19. To our knowledge, this is the first time that such logarithmic time dependencies of $R^{2}(t)$ are predicted for a model aimed to describe a disordered system such as porous Silicon. Actually, the value of the exponent describing the logarithmic time dependence turns out to depend slightly on temperature, and the value of four is observed only for $T \cong 30 \mathrm{~K}$. At lower $T, R$ tends to extrapolate to a constant value because non-radiative annihilation events become dominant, and the carriers can only explore a finite spatial extent asymptotically. A similar trend is also observed for electrons and holes in the case of uncorrelated electron-hole pairs. 


\section{TWO- AND THREE-DIMENSIONAL MODELS AND RESULTS}

The actual geometrical structure of porous Silicon is not known accurately. For many purposes, however, one may hope to capture the essential features of such complex structures by modeling them with simple, yet non trivial, percolation-like clusters. [36] The clusters are generated by using a modified version of the well-known growth algorithm employed for percolation clusters (see e.g. Ref. [36]), which is adapted here to our present purposes.

For simplicity we consider a square lattice in two dimensions, and a simple cubic one in three dimensions. The linear size of the lattice is denoted by $\ell$. The growing process starts at the seed, which is located at the center of the otherwise empty lattice. The growth proceeds according to the following rules. A nearest neighbor site $j$ of the seed can be occupied with probability $p_{n}$, with the index $n$ indicating the number of occupied sites nearest to the growing site $j$. Initially, $n=1$. If the site $j$ is not occupied, i.e. if it does not become part of the cluster, it is blocked and cannot be occupied later. The process continues from the last occupied sites and now values $n>1$ may occur.

For standard percolation clusters, one takes $p_{n}=p$ independently of $n$. When $p<p_{\text {crit }}$, $p_{\text {crit }} \cong 0.593$ (two dimensions), and $p_{\text {crit }} \cong 0.312$ (three dimensions), only finite clusters can grow, while infinite clusters develop when $p>p_{\text {crit }}$. When $p=p_{\text {crit }}$, large percolation clusters may grow which are fractal (with fractal dimension $d_{f} \cong 1.896$ in two dimensions, and $d_{f} \cong 2.5$ in three dimensions) on large length scales. Finite clusters and the infinite cluster above $p_{\text {crit }}$ are also fractal (with the same fractal dimension $d_{f}$ ) for length scales smaller than the correlation length. The latter diverges at $p=p_{\text {crit }}$.

In our model, we take $p_{1}>p_{2}$ and $p_{n}=0$ when $n>2$. In the following, we consider the cases, $p_{1}=0.65$ and $p_{2}=0.15$ in two dimensions, and $p_{1}=0.45$ and $p_{2}=0.17$ in three dimensions.

We notice that when $p_{1} \rightarrow 1$, and $p_{2} \rightarrow 0$, the clusters tend to grow linearly, while for relatively large $p_{2}$, compact clusters can be grown. By varying both $p_{1}$ and $p_{2}$, a variety of structures can be obtained which are suitable for our present purposes. A measure of the 
porosity of the cluster is simply given by the ratio between empty sites and occupied sites. Typical examples are shown in Fig. 15 for three dimensional clusters, and in Ref. [20] for two-dimensional clusters. The clusters are uniform on large length scales, but still display spatial fluctuations on small length scales as the infinite cluster above $p_{\text {crit }}$. 20] Thus, the intrinsic fractal character of percolation is common to the clusters shown in Fig. 15 too. It is possible to construct highly constrained and filamentary clusters (like that in Fig. 15(a)) or more compact clusters (like that in Fig. 15(b)).

We have studied the dynamical behavior of carriers on these clusters, following the same MC-rules described above. The question now is how the extra degrees of freedom in space, resulting from the two- or three-dimensional topology of the clusters, modify the time decay of $L(t)$. Preliminary results for two-dimensional systems have been reported in Ref. [40]. Results for three-dimensional systems are reported in Fig. 16 for the luminescence decay. [20] For excitons and uncorrelated electron-hole pairs, the lineshape is a stretched exponential and a power law, respectively. Hence the decay lineshape is not modified by the increased dimensionality. Results of stretched exponential fits to the luminescence decay in two or three dimensions, for the exciton model, are reported in Fig. 10 as a function of temperature.

The three-dimensional topology has important consequences on the decay of the luminescence. The $\tau$ and $\beta$ values are lower, indicating that the role of hopping processes in three dimensions is more important than in lower dimensions. While the one-dimensional model (and also the two-dimensional one) predicts values of $\beta$ close to unity already at room temperature, in three dimensions, in contrast, the theoretical values are consistent with the experimental results for $\beta$, which typically saturate at values $\beta \cong 0.7-0.8$. [18, 19] Thus, values of $\beta<1$ at high temperatures, and intermediate porosities, can be explained by our model as a result of the interplay between a complex conducting matrix (representing the topology of p-Si), and an additional local disorder due to the distribution of nano-crystal sizes in the system.

A comparison of the temperature dependence of $\tau$ and $\beta$ for two different sets of pa- 
rameters (but for the same growth parameters $p_{1}, p_{2}$ mentioned above) is reported in Fig. 17. The effect of variing $\tau_{\mathrm{nr}}$ is evident in $\beta$ : the larger the $\tau_{\mathrm{nr}}$ the lower is $\beta$. The role of temporary traps, which tend to reduce $\beta$ and are effective at low temperatures, is enhanced when $\tau_{\text {nr }}$ is increased due to a relative increase of the role of exciton diffusion $\left(\sim \tau_{\text {nr }} / \tau_{\text {hop }}\right)$.

The dynamics of carriers is influenced by the parameters $p_{1}$ and $\mathrm{p}_{2}$ used to construct the clusters. The more the cluster is filamentary (see e.g. case (a) in Fig. 15), the more the excitons are constrained to move along one direction, and the larger are $\tau$ and $\beta$. Then, the dynamical behavior of carriers should have essentially a one-dimensional character, at least for sufficiently low temperatures where transport along the structure is strongly hindered. Thus, we expect to observe, in this regime, the same logarithmic time dependence of $R^{2}(t)$ as in the one-dimensional model, Eq. 19, at short times.

For arbitrary porosities and not too low temperatures, we find anomalous behavior of $R^{2}(t) \sim t^{2 / d_{w}}$, at intermediate times (Fig. 18). Here, $d_{w}>2$ and increases as the temperature decreases, more than in the one-dimensional case (Fig. 12). Eventually, a logarithmic time dependence of $R^{2}(t)$ is observed for sufficiently low $T$. In three dimensions, we have found clear evidence for the behavior

$$
R^{2} \sim(\log t)^{2}
$$

at long times (Fig. 19), in contrast to the fourth-power result valid for the Sinai model. This new behavior represented by Eq. 20 has been predicted for the case of biased diffusion on random fractals in two dimensions. [39] As we can see, a similar behavior can be expected also in three dimensions. This feature remains to be studied theoretically.

\section{DISCUSSION}

According to the present model, the photoluminescence decay, $\mathrm{L}(\mathrm{t})$, is determined, in the case of excitons, by the recombination and the escape rates from the isolated QD. In the case of uncorrelated electron-hole pairs, $\mathrm{L}(\mathrm{t})$ is determined by the diffusion of the electron and the hole, because both need to be on the same QD to recombine radiatively. 
In the case of excitons, the disordered environments of the QDs, from which the luminescence is observed, cause a distribution of waiting times for hopping and/or release times for activated emission from the temporary traps, and the luminescence displays a stretched exponential decay. In the case of uncorrelated electron-hole pairs, the main role is played by the encounter probability of the electron and the hole to be on the same site. This is determined by the diffusion of the slower moving particle, i.e. the hole, and the time dependence of the recombination rate becomes a power-law, [23] which is then reflected in the luminescence decay.

For all spatial dimensions $D$, the increase in $\beta$ is almost linear for $10<\mathrm{T}<60 \mathrm{~K}$, has a plateau for $60<\mathrm{T} \leq 200 \mathrm{~K}$ and then increases rapidly to 1 at higher temperatures. This behavior corresponds to the following three typical regimes: a first one in which the temporary traps play a role (low temperatures), a second one in which hopping dominates and diffusion is restricted only by the p-Si network geometry (intermediate temperatures), and a third one, in which the rapid on-site non-radiative recombinations are most effective and dominant (high temperatures).

Whereas there is general agreement among different experimentalists that $\tau$ strongly depends on temperature, $[7,8,[8]$ the situation is less clear regarding the exponent $\beta$. While some authors report values of $\beta \cong 0.8-0.9$, independent of the temperature [15, 16], others found temperature dependent values. [17 [19] In this case, the $\beta$ values follow similar temperature and energy dependences in the range 0.4-0.8. By varying the parameters in our model, we are able to describe both situations. Temperature dependent $\beta$ values are shown in Fig. 17. Temperature independent values can be obtained when $\tau_{\text {hop }}<\tau_{\text {nr }} a n d \tau_{\text {sing }}$, since in this case the activation term in Eq. 10, which is mainly responsable for the temperature dependence of $\beta$, does not influence the diffusion of carriers, being only restricted by the geometrical constraints of the p-Si network.

By going from an interconnected array of QDs to an ensemble of isolated QDs our model predicts that the lifetime $\tau$ and the dispersion exponent $\beta$ increase, with $\beta$ taking values near 1. This has been indeed observed in several experiments. For example, the data presented in 
Ref. [41], and reanalised using stretched exponential functions, show that $\tau$ and $\beta$ increase after dry oxidation of p-Si. It is well know that the interconnected array of QDs typical of p-Si is transformed after dry oxidation into a dispersion of nano-crystals immersed in a $\mathrm{SiO}_{x}$ matrix; increasing further the oxidation a porous glass is formed. [6]

Our simulations show that hopping of excitons is responsable for the stretched exponential decay observed in p-Si. This rules out other explanations which are based on analogies with hydrogenated amorphous $\mathrm{Si}(\mathrm{a}-\mathrm{Si}: \mathrm{H})$, see e.g. Ref. 42. In fact we demonstrated that PL decay lineshapes, strongly resembling those measured in a-Si:H, are found for uncorrelated e-h pairs. This behavior can possibly explain the lack of room temperature luminescence in a-Si:H. In fact, it is possible that the fast diffusing species in the pair is rapidly trapped into non-radiative recombination centers when the thermal energy is sufficiently large to promote the particle to extended states. 443

Annealing treatments induce a quenching of the room temperature luminescence with a decrease in the values of $\tau$ and $\beta$. [14] Within our model, this is simulated by diminishing $\tau_{\mathrm{nr}}^{(0)}$ and $\tau_{\text {hop }}$ yielding lower values of $\tau, \beta$ and quantum efficiency (see Table II). The separate effects of variing $\tau_{\text {nr }}^{(0)}$ and $\tau_{\text {hop }}$ are the following:

(a) Reducing the non-radiative lifetime, yields an increase of $\beta$ and a decrease of $\tau$. The reason is that the re-population of the target QDs, those from which the luminescence decay is recorded, is reduced by the competitive non-radiative recombination channels. Less excitons reach the target QD at long times because a large part recombine non-radiatively as they diffuse through the system. The target QD effectively behaves as being more isolated (increase of $\beta$ ). Then, $\tau$ is reduced due to the competing on-site non-radiative recombinations, and consequently the quantum efficiency is also reduced.

(b) Reducing $\tau_{\text {hop }}$ yields a reduction of $\tau$ and $\beta$. In fact, for higher hopping probabilities, the target QDs are emptied faster due to the competing loss of excitons caused by hopping (reducing $\tau$ ), while at long times more excitons reach the target QD giving rise to longlived recombinations (reducing $\beta$ ). In this case, no variations in the quantum efficiency are expected. 
Hence to obtain the trends measured during the annealing experiments both the lifetimes, $\tau_{\mathrm{nr}}^{(0)}$ and $\tau_{\text {hop }}$, should be reduced.

Let us compare our simulations with other models. The non-exponential decay of $\mathrm{L}(\mathrm{t})$ has been explained as due to a distribution of $\tau_{\text {rad }}$ values arising from a shape distribution of quantum dots with the same emission energy. [7] Within this assumption, however, it is difficult to explain the temperature dependence of $\beta$ already discussed. It has also been proposed that the non-exponential behavior of $\mathrm{L}(\mathrm{t})$ is a consequence of a distribution of nonradiative decay rates. 44,45 This fact is not considered in our model, and a refined version of it should certainly contain such a dependence. However, we want to emphasize here that it is the dispersive motion of excitons which is mostly responsible for the non-exponential decay of $\mathrm{L}(\mathrm{t})$. The radiative and non-radiative lifetime distributions could indeed concur to produce a similar effect on $\mathrm{L}(\mathrm{t})$, but in isolated and well passivated quantum dots it is reasonable to expect essentially a simple exponential decay of $\mathrm{L}(\mathrm{t})$. Such a behavior has been indeed observed for some p-Si samples. 46]

The strong temperature dependence of the effective anomalous diffusion exponent $d_{w}$, and the logarithmic time dependencies of $R^{2}(t)$ at lower temperatures, have important consequences on the ac-conductivity, which is expected to display a strong frequency dependence at low temperatures. This observation is supported by recent experimental results on the ac-conductivity of p-Si. 477 In contrast, at higher temperatures, diffusion becomes normal, $R^{2}(t) \sim t$, i.e. $d_{w} \rightarrow 2$, and the dispersion frequency range shrinks considerably. It should be emphasized that a model for the structure of p-Si has been suggested recently, [47 in which the p-Si network is assumed to be the infinite percolation above criticality. From our present results, however, one can see that such a model is far too simple to describe p-Si samples of different porosities, and a more elaborated model is required. In addition, theoretical results for the ac-conductivity discussed so far, [47 have been obtained using mean-field approximations, and the new intrinsic behavior suggested here for diffusion of carriers in p-Si at low temperatures can not be obtained. 


\section{CONCLUSION}

The theoretical results obtained in this paper have for the moment a semi-quantitative character. A quantitative comparison between the present results and the available experimental data can be made possible when the parameters entering the model may be estimated independently and more accurately. Also, the precise relations between the nanocrystal structure and dynamical properties are so far unkown. We have tried to fill this lackness by making ad hoc, yet standard assumptions which seem to describe the physics of p-Si rather well. In addition, the actual values of the parameters are so strongly sample dependent and influenced by various treatments (ageing, oxidation, storage, excitation conditions, etc.) that quantitative predictions valid in general are not possible. However, several experimentally measured trends in the photoluminescence lifetimes, dispersion exponents and quantum efficiencies of the luminescence are correctly explained in the frame of the present calculations by choosing the free parameters of the model appropriately. This gives a strong support for the validity of the present model for describing the recombination dynamics in p-Si.

Finally, by assuming different forms for the various quantities reported in Section II, other systems can be described as well by the present model, as e.g. nanometer sized Si crystallites, [48] CdSSe quantum dots, [49] etc. All these systems show a stretched exponential decay of the luminescence. 


\section{REFERENCES}

* Corresponding author; email: pavesi@science.unitn.it, fax: +39/461/881696.

[1] L. T. Canham, Appl. Phys. Lett. 57, 1046 (1990).

[2] (a) Porous Silicon, edited by Zhe Chuan Feng and R. Tsu (World Scientific Publishing Co., New York 1995); (b) Porous Silicon Science and Technology, edited by J.-C. Vial and J. Derrien (Les Editions de Physique, Les Ulis 1995).

[3] Optical Properties of Low Dimensional Silicon Structures, edited by D. C. Benshael, L. T. Canham and S. Ossicini, NATO ASI Series vol. 244 (Kluwer Academic Publisher, Dordrecht 1993).

[4] Light Emission from Silicon, special issue of J. of Lumin. 57 (1993); Porous Silicon and Related Materials, special issue of Thin Solid Films 255 (1995).

[5] R. L. Smith and S. D. Collins, J. Appl. Phys. 71, R1 (1992).

[6] L. T. Canham, in Ref. [3] pag. 81.

[7] P. D. J. Calcott, K. J. Nash, L. T. Canham, M. J. Kane and D. Brumhead, J. Phys.: Condens. Matt. 5, L91 (1993); P. D. J. Calcott, K. J. Nash, L. T. Canham, M. J. Kane and D. Brumhead, J. of Lumin. 57, 257 (1993).

[8] J. C. Vial, A. Bsiesy, G. Fishman, F. Gaspard, R. Herino, M. Ligeon, F. Muller, R. Romestain, and R. M. Macfarlane, in Microcrystalline Semiconductors: Materials Science and Devices, ed. by Y. Aoyagi, L. T. Canham, P. M. Fauchet, I. Shimizu and C. C. Tsu, Mat. Res. Symp. Proc. vol 283 (1993) pag. 241.

[9] F. Koch, Mat. Res. Symp. Proc 298, 319 (1993); F. Koch, V. Petrova-Koch, and T. Muschik, J. of Lumin. 57, 271 (1993); F. Koch and V. Petrova-Koch, The surface state mechanism for light emission from porous Silicon, in Ref. [2] a, pag. 133.

[10] S. M. Prokes, O. J. Glembocki, V. N. Bermudex, R. Kapla, L. E. Friedersdorf, and P. 
C. Searson, Phys. Rev. B 45, 13788 (1992).

[11] M. S. Brandt, H. D. Fuchs, M. Stutzmann, J. Weber, and M. Cardona, Solid State Commun. 81, 307 (1992).

[12] J. M. Lavine, S. P. Sawan, Y. T. Shieh, and A. J. Bellezza, Appl. Phys. Lett. 62, 1099 (1993).

[13] X. Chen, B. Henderson, and K. P. O’Donnel, Appl. Phys. Lett. 60, 2672 (1992).

[14] N. Ookubo, H. Ono, Y. Ochiai, Y. Mochizuki, and S. Matsui, Appl. Phys. Lett. 61, 940 (1992).

[15] M. Kondo, J Non-Crystalline Sol. 164-166, 941 (1993).

[16] Y. Kanemitsu, Phys. Rev. B 48, 12357 (1993).

[17] N. Ookubo, N. Hamada and S. Sawada, Solid St. Commun. 92, 369 (1994).

[18] L. Pavesi and M. Ceschini, Phys. Rev. B48, 17625 (1993).

[19] L. Pavesi, M. Ceschini, and H. E. Roman, Thin Solid Films 255, 67 (1995).

[20] L. Pavesi and H. E. Roman, in Microcrystalline and Nanocrystalline Semiconductors, edited by L. Brus, R. W. Collins, M. Hirose and F. Koch, Materials Research Society Symposium Proceedings Series 358 (1995).

[21] G. Pfister and H. Scher, Adv. Phys. 27, 747 (1978).

[22] J. Klafter and M. F. Shlesinger, Proc. Natl. Acad. Sci. USA 83, 848 (1986).

[23] H. Scher, M. F. Shlesinger and J. T. Bendler, Physics Today 44, 26 (January 1991).

[24] Ph. Dumas, et al., Europhys. Lett. 23, 197 (1993).

[25] A generic quantity $\mathrm{X}$, representing for instance the particle size $d$, is labeled with an index $i, X_{i}$, to indicate its actual local value at the $i$-th site. 
[26] C. Delerue, M. Lannoo, and G. Allan, J. of Lumin. 57, 249 (1993).

[27] A. D. Yoffe, Advances in Physics 42, 173 (1993).

[28] M.S. Hybertsen, Phys. Rev. Lett. 75, 1514 (1994).

[29] G. Fishman, R. Romestain, and J.C. Vial, J. of Lumin. 57, 235 (1993).

[30] B.K. Ridley, J. Phys. C11, 2323 (1978).

[31] M. Rosenbauer, M. Stutzmann, H.D. Fuchs, S. Finkbeiner, and J. Weber, J. of Lumin. 57, 153 (1993).

[32] E. Bustarret, M. Ligeon, I. Mihalcescu, J. Oswald, Thin Solid Films 255, 234 (1995).

[33] O. Teschke, M. C. Goncalves and F. Galembeck, Appl. Phys. Lett. 63, 1348 (1993).

[34] J. Noolandi, K. M. Hong, and R. A. Street, Solid St Commun. 34, 45 (1980).

[35] R. A. Street, Advances in Physics 30, 593 (1981).

[36] A. Bunde and S. Havlin, eds., Fractals and disordered systems (Springer Verlag, Heidelberg, 1991).

[37] M. Silver, G. Schoenherr and H. Baessler, Phys. Rev. Lett. 48, 352 (1982).

[38] Y. Sinai, Theor. Prob. Appl. 27, 256 (1892).

[39] H.E. Roman, M. Schwartz, A. Bunde and S. Havlin, Europhys. Lett. 7, 389 (1988).

[40] M. Ceschini and L. Pavesi, in Proceedings of the 22th International Conference on the Physics of Semiconductors, ed. D. J. Lockwood ( World Scientific Publishing Co, 1995) pag. 2165.

[41] M. Yamada, A. Takazawa, and T. Tamura, Jpn. J. Appl. Phys. 31, L1451 (1992).

[42] L. R. Tessler, F. Alvarez, and O. Teschke, Appl. Phys. Lett. 62, 2381 (1993).

[43] L. Pavesi, Luminescence of porous and amorphous hydrogenated Silicon: analogies and 
differences, in Amorphous Hydrogenated Silicon, Solid State Phenomena:part B Diffusion and Defect Data (Scitec Publications Ltd. Zug, 1995).

[44] T. Suemoto, K. Tanaka and A. Nakajima, Phys. Rev. B 49, 11005 (1994).

[45] S. Sawada, N. Hamada, and N. Ookubo, Phys. Rev. B 49, 5236 (1994).

[46] G. W. 't Hooft, Y. A. R. R. Kessner, G. L. J. A. Rikken, and A. H. J. Venhuizen, Appl. Phys. Lett. 61, 2344 (1992).

[47] M. Ben-Chorin, F. Moller, F. Koch, J. of Lumin. 57, 19 (1993); M. Ben-Chorin, F. Moller, F. Koch, W. Schirmacher, and M. Eberhard, Phys. Rev. 51, 2199 (1995).

[48] Y. Kanemitsu, Phys. Rev. B 49, 16845 (1994).

[49] A. S. Dissanayake, G. W. Wen, W. Hein, J. Y. Lin, and H. X. Jiang, Proceedings of the 22th International Conference on the Physics of Semiconductors, ed. D. J. Lockwood (World Scientific Publishing Co, 1995) pag. 1827. 


\section{TABLES}

TABLE I. Values used in the Monte-Carlo calculations. $\ell^{(3 / D)}$ is the linear size of the $D$-dimensional lattice employed, $\mathrm{t}_{\max }$ the maximum number of $\mathrm{MC}$ step for a single run, $\mathrm{n}_{\text {runs }}$ the number of runs (i. e. the number of different particles used) for a single cluster realization, $\mathrm{n}_{\text {conf }}$ the number of different cluster realizations over which the reported results are averaged. The meaning of the other parameters is given in the text. All the results presented in this paper refer to this choice of parameters unless otherwise stated.

\begin{tabular}{cccccccccccc}
\hline \hline$\ell$ & $\mathrm{t}_{\max }$ & $\mathrm{n}_{\text {runs }}$ & $\mathrm{n}_{\text {conf }}$ & $\tau_{\text {hop }}$ & $\tau_{\text {sing }}^{(0)}$ & $\mathrm{n}_{s}$ & $\tau_{\mathrm{nr}}^{(0)}$ & $\hbar \omega_{\mathrm{ph}} / k$ & $\mathrm{p}$ & Porosity & $\mathrm{E}_{\text {cut }}$ \\
31 & 4000 & 2000 & 200 & $150 \mu \mathrm{s}$ & $150 \mu \mathrm{s}$ & 1.5 & $900 \mu \mathrm{s}$ & $800 \mathrm{~K}$ & 25 & $65 \%$ & $0.04 \mathrm{eV}$ \\
\hline \hline
\end{tabular}

TABLE II. $300 \mathrm{~K}$ simulation results for a $3 \mathrm{D}$ clusters of $65 \%$ porosity as a function of the time parameters: $\tau_{\mathrm{nr}}^{(0)}$ and $\tau_{\text {hop }}$. The other parameters are given in Table I.

\begin{tabular}{llccc}
\hline \hline$\tau_{\text {nr }}^{(0)}$ & $\tau_{\text {hop }}$ & $\tau$ & $\beta$ & QE \\
$(\mu \mathrm{s})$ & $(\mu \mathrm{s})$ & $(\mu \mathrm{s})$ & & $\%$ \\
\hline 900 & 150 & 72 & 0.77 & 12 \\
800 & 300 & 84 & 0.87 & 10 \\
800 & 100 & 59 & 0.72 & 10 \\
500 & 150 & 51 & 0.84 & 7 \\
\hline \hline
\end{tabular}




\section{FIGURES}

FIG. 1. Distribution of optical-band gaps for an ensemble of quantum dots (dotted line) (Eq. 2), and corresponding density of states (continuous line) (Eq. 4) versus energy.

FIG. 2. Temperature dependence of the transition times (Eq. 13) for an energy of $1.86 \mathrm{eV}: \tau_{\text {rad }}$ (short-dashed lines), $\tau_{\mathrm{nr}}$ (long-dashed lines) and $\tau$ (continuous line). Here we have used the same set of parameters as given in Table VI except for: case (1), thick lines: $\tau_{\text {sing }}^{(0)}=150 \mu \mathrm{s}, \tau_{\mathrm{nr}}^{(0)}=900 \mu \mathrm{s}$;

case $(2)$, thin lines: $\tau_{\text {sing }}^{(0)}=100 \mu \mathrm{s}, \tau_{\mathrm{nr}}^{(0)}=400 \mu \mathrm{s}$; and case $(3)$, thin lines: $\tau_{\text {sing }}^{(0)}=200 \mu \mathrm{s}$, $\tau_{\mathrm{nr}}^{(0)}=7000 \mu \mathrm{s}$.

FIG. 3. Quantum efficiency of an ensemble of isolated quantum dots: $\epsilon(E, T) P(E)$ versus $E$ for various temperatures reported on the curves in K. The temperature dependence of the Si band-gap has been neglected.

FIG. 4. Total quantum efficiency of the ensemble of quantum dots, $\epsilon(T)$, versus temperature. Here we have used the same set of parameters as given in Table VI except for: case (1), thick line: $\tau_{\text {sing }}^{(0)}=150 \mu \mathrm{s}, \tau_{\mathrm{nr}}^{(0)}=900 \mu \mathrm{s} ;$ case $(2)$, thin line: $\tau_{\text {sing }}^{(0)}=100 \mu \mathrm{s}, \tau_{\mathrm{nr}}^{(0)}=400 \mu \mathrm{s}$; and case $(3)$, thin dashed line: $\tau_{\text {sing }}^{(0)}=200 \mu \mathrm{s}, \tau_{\mathrm{nr}}^{(0)}=7000 \mu \mathrm{s}$. The thick dashed lines represent the exponential behavior $\epsilon(T) \sim \exp \left(-T / T_{0}\right)$, with $T_{0}=59 \mathrm{~K}$ for case (1) and case (2) and $T_{0}=75 \mathrm{~K}$ for case (3).

FIG. 5. Absorption coefficient of the ensemble of isolated quantum dots, $\alpha(E, T)$, versus energy for the indicated temperatures, $\mathrm{T}$.

FIG. 6. Snapshot of the energy landscape for diffusion in a one-dimensional lattice. The transition energy $E$ is plotted versus position, for: $E_{\text {cut }}=\infty$ (uncorrelated values, top panel), $0.04 \mathrm{eV}$ (middle panel), and $0.004 \mathrm{eV}$ (bottom panel). The mean energy value over the 29791 sites is 1.86 $\mathrm{eV}$ for the three cases.

FIG. 7. Monte-Carlo simulation results for a one-dimensional system of excitons. Luminescence versus time for four different temperatures: $T=20 \mathrm{~K}$ (squares), $100 \mathrm{~K}$ (triangles), $300 \mathrm{~K}$ (discs) and $500 \mathrm{~K}$ (empty circles). 
FIG. 8. Fitting of the luminescence decay (circles) obtained from a Monte-Carlo simulation for a one-dimensional system of excitons at $T=100 \mathrm{~K}$. The following fitting functions and best fit parameters have been used: Stretched exponential function with $\tau=345 \mu$ s and $\beta=0.815$ (full line); single exponential decay with $\tau=555 \mu$ s (dotted line); double exponential with $\tau_{1}=80 \mu \mathrm{s}$ and $\tau_{2}=503 \mu \mathrm{s}$ (dashed-dotted line). The top panel reports the relative errors, defined as the ratio of the difference of the simulation data $(Y)$ minus the computed value $\left(Y_{F I T}\right)$ times the simulation data, i. e. $\left[\left(Y-Y_{F I T}\right) / Y\right]$, for the three fitting functions.

FIG. 9. Monte-Carlo simulation results for a one-dimensional system of uncorrelated electron and hole pairs. Luminescence versus time for four different temperatures: $T=30 \mathrm{~K}$ (dots), $50 \mathrm{~K}$ (circles), $100 \mathrm{~K}$ (triangles) and $300 \mathrm{~K}$ (squares). The lines are power-law fits to the decay with the following exponents: $\alpha=0.86(T=30 \mathrm{~K}), 0.79(T=50 \mathrm{~K}), 0.73(T=100 \mathrm{~K})$ and $0.91(T=300$ K). The curves are vertically shifted for clarity.

FIG. 10. Temperature dependence of the luminescence recombination time $\tau$ (empty discs) and dispersion exponent $\beta$ (filled discs) obtained by a stretched exponential fit to the time decay of the luminescence, for an exciton population in one-dimensional (upper panel), two-dimensional (middle panel) and three dimensional (lower panel) clusters.

FIG. 11. Mean square displacement $R^{2}(t)$ of excitons in one dimension for three different temperatures: $T=20 \mathrm{~K}$ (circles), $100 \mathrm{~K}$ (triangles) and $500 \mathrm{~K}$ (squares). Note the departure from a power-law behavior at low temperatures. The curves are vertically shifted for clarity.

FIG. 12. Anomalous diffusion exponent $d_{w}$ obtained by a power-law fit to the mean square displacement $R^{2}(t)$ of excitons in one-dimensional (circles), two-dimensional (discs) and three-dimensional (triangles) clusters. 
FIG. 13. Energy landscape (transition energies $E$ vs position) in one dimension. The arrows are drawn in direct correspondence with the local energy difference between nearby sites. The resulting one-dimensional chain (lower panel) is equivalent to the Sinai model, in which uncorrelated random fields are present at each site of the lattice.

FIG. 14. Mean square displacement $R^{2}(t)$ of excitons in one dimension plotted versus $(\log t)^{4}$ for the temperatures: $T=20 \mathrm{~K}, 30 \mathrm{~K}$ and $50 \mathrm{~K}$. The straight line through the $T=30 \mathrm{~K}$ results represents the asymptotic $(\log t)^{4}$ behavior. The line through the $T=50 \mathrm{~K}$ results shows the power law $t^{2 / d_{w}}$ behavior, with $d_{w}=2.5$.

FIG. 15. Percolation-like clusters grown on a simple cubic lattice representing porous Silicon of the same $97.4 \%$ porosity but different growth probabilities: (a) $p_{1}=0.9, p_{2}=0.05035$, (b) $p_{1}=0.3235, p_{2}=0.4$.

FIG. 16. Luminescence decay of a three-dimensional system of excitons (upper panel) and of uncorrelated electron-hole pairs (lower panel) for three different temperatures: $T=300 \mathrm{~K}$ (circles), $200 \mathrm{~K}$ (discs) and $100 \mathrm{~K}$ (triangles). The lines are fits to the luminescence decays. Stretched exponentials have been used for the exciton case with the following parameters: $\tau=175 \mu$ s and $\beta=0.58(T=100 \mathrm{~K}), \tau=135 \mu \mathrm{s}$ and $\beta=0.62(T=200 \mathrm{~K})$, and $\tau=66 \mu \mathrm{s}$ and $\beta=0.73$ $(T=300 \mathrm{~K})$. Power laws, $t^{-\alpha}$, have been used for the uncorrelated electron-hole pairs with the following parameters: $\alpha=0.96(T=100 \mathrm{~K}), \alpha=1.10(T=200 \mathrm{~K})$ and $\alpha=1.28(T=300 \mathrm{~K})$. In this case, the curves are vertically shifted for clarity.

FIG. 17. Temperature dependence of the luminescence recombination times $\tau$ (empty symbols) and dispersion exponents $\beta$ (filled symbols) for excitons in three dimensional clusters and for two different sets of parameters. Circles correspond to case (1) of Fig. 2 and to the standard set of parameters reported in the Table $\mathrm{VI}$. Triangles correspond to case (2) of Fig. 2 and hopping time $\tau_{\text {hop }}=100 \mu \mathrm{s}$. The lines are the lifetimes calculated using Eq. 13 in the case of isolated quantum dots, i. e. without hopping, for case (1) (full line) and case (2) (dotted line). 
FIG. 18. Mean square displacement $R^{2}(t)$ of excitons in three dimensional clusters for four different temperatures: $T=20 \mathrm{~K}$ (squares), $T=30 \mathrm{~K}$ (triangles), $T=50 \mathrm{~K}$ (circles) and $T=100$ K (discs). The curves are vertically shifted for clarity. The lines are power law fits. Note, however, the bending of the data at low temperatures for short times.

FIG. 19. Mean square displacement $R^{2}(t)$ of excitons in three dimensional clusters for the temperature $T=30 \mathrm{~K}$, plotted as a function of $(\log t)^{2}$. Note the linear increase at long times. 